\title{
Magnetic dipole excitation and its sum rule in nuclei with two valence nucleons
}

\author{
Tomohiro Oishi ${ }^{1, *}$ and Nils Paar ${ }^{1, \dagger}$ \\ ${ }^{1}$ Department of Physics, Faculty of Science, University of Zagreb, Bijenička c. 32, 10000 Zagreb, Croatia
}

\begin{abstract}
Background: Magnetic dipole (M1) excitation is the leading mode of nuclear excitation by the magnetic field, which couples unnatural-parity states. Since the M1 excitation occurs mainly for open-shell nuclei, the nuclear pairing effect is expected to play a role. As expected from the form of operator, this mode may provide the information on the spin-related properties, including the spin component of dineutron and diproton correlations. In general, the sum rule for M1 transition strength has not been derived yet.

Purpose: To investigate the M1 excitation of the systems with two valence nucleons above the closed-shell core, with pairing correlation included, and to establish the M1 sum rule that could be used to validate theoretical and experimental approaches. Possibility to utilize the M1 excitation as a tool to investigate the pairing correlation in medium is also discussed.

Method: Three-body model, which consists of a rigid spherical core and two valence nucleons, is employed. Interactions for its two-body subsystems are phenomenologically determined in order to reproduce the two-body and three-body energies. We also derive the M1 sum rule within this three-body picture.

Conclusion: The introduced M1 sum rule can be utilized as a benchmark for model calculations of M1 transitions in the systems with two valence nucleons. The total sum of the M1 transition strength is related with the coupled spin of valence nucleons in the open shell, where the pairing correlation is unnegligible. The three-body-model calculations for ${ }^{18} \mathrm{O},{ }^{18} \mathrm{Ne}$, and ${ }^{42} \mathrm{Ca}$ nuclei demonstrate a significant effect of the pairing correlations on the low-lying M1 transitions. Therefore, further experimental studies of M1 transitions in those systems are on demand, in order to validate proposed sum rule, provide a suitable probe for the nuclear pairing in medium, as well as to optimize the pairing models.
\end{abstract}

PACS numbers: 21.10.-k, 21.45.+v, 23.20.-g, 23.20.Lv

\section{INTRODUCTION}

Electromagnetic excitations in finite nuclei are fundamental in nuclear physics and astrophysics. They provide valuable probe of the nuclear structure and dynamics and play a decisive role in the processes in stellar environments. In particular, magnetic dipole (M1) transitions have invoked various interests and discussions in recent studies [1-4]. The M1 excitation mode is relevant to a diversity of nuclear properties, including unnaturalparity states, spin-orbit splitting, tensor force effect, etc. Several collective nuclear phenomena, including scissors mode in deformed nuclei, can be activated by the M1 excitation [5-14]. In addition, the correspondence between the M1 and the zeroth component of Gamow-Teller modes has been discussed [10, 15, 16]: the M1 excitation can be utilized to optimize theoretical methods to predict the Gamow-Teller resonance distribution, which plays an essential role in neutrino-nucleus scattering.

Within a shell-model picture, the M1 transition couples spin-orbit partner orbits. Thus, this process is measurable mainly in open-shell nuclei, where the transition from the lower to higher partner orbit is available [57, 17-23]. In such systems, nuclear pairing correlation can play an essential role [24-27]. However, compared

\footnotetext{
* E-mail: toishi@phy.hr

† E-mail: npaar@phy.hr
}

with the electric dipole (E1) and quadrupole (E2) modes [28-34], the knowledge on the pairing effect on magnetic modes, as well as on unnatural-parity states, is rather limited [10-12, 20].

In studies of nuclear modes of excitation, the sum rules associated to the transition strength and energyweighted sum rules, represent an essential tool for the analyses of the excitations, not only as benchmark tests of the theoretical frameworks involved, but also to inspect the completeness of the experimental data [35-48]. Over the past decades, the analyses of the sum rules have been mandatory to validate theoretical approaches to describe various modes of excitation. In particular, the Fermi sum rule and Gamow-Teller sum rules are well established for the isospin-flip and spin-flip isospinflip charge-exchange resonances, respectively [39, 40, 49]. The sum rules for electric multipole modes have also been established and very useful in studies of giant monopole, dipole and quadrupole resonances [35-37]. Recently, the general formulas have been derived for non-energyweighted and energy-weighted sum rules of electric and weak transitions within a shell model occupation-space framework [38]. However, so far, the general version of sum rule has not been established for the M1 excitation. It represents an open problem of relevance for complete understanding of magnetic transitions.

In this work, we explore the properties of M1 excitations with a strong pairing, by employing the three-body model. This model is applicable to nuclei composed of the closed-shell core and two valence nucleons. In this 
model assumption, the sum rule of M1 transition is also introduced. Its implementation for the purpose of the present study may provide a standard to benchmark the theoretical investigation to some nuclei, where the threebody picture can be a good approximation.

It is worthwhile to mention so-called dineutron and diproton correlations, which can be relevant to our interest. Several theoretical studies have predicted that, under the effect of the pairing correlation in medium, the valence two-nucleon subsystem may have a spin-singlet $\left(S_{12}=0\right)$ and/or spatially localized structure $[28,50$ 56]. However, considering its measurability, there remain some problems to clarify this intrinsic structure [57-59]. As expected from the form of the M1 transition operator, it may provide a suitable way to probe the coupled spin of nucleons, $S_{12}$, which should reflect the pairing effect inside nuclei.

Our theoretical formalism, as well as numerical methods, are described in section II. Then, section III is devoted to present our results and discussion. Finally, the summary and conclusion of this work are given in section IV. The CGS-Gauss system of units is used in this article.

\section{THREE-BODY MODEL}

We employ the three-body model, which has been developed in Refs. [28, 52-56, 60-63]. Namely, the system consists of a rigid-core nucleus and two valence nucleons with the following assumptions.

- Core nucleus is spherical, i.e., with the shell-closure and $J^{\pi}=0^{+}$. Nucleons in the core are not active for excitation.

- Two valence nucleons are of the same kind, namely, protons or neutrons. They feel the mean field, $V_{C}$, generated by the core nucleus.

Thus, the three-body Hamiltonian reads

$$
\begin{gathered}
H=h_{C}(1)+h_{C}(2)+v_{N N}\left(\boldsymbol{r}_{1}, \boldsymbol{r}_{2}\right)+x_{\mathrm{rec}} \\
h_{C}(i)=\frac{p_{i}^{2}}{2 \mu_{i}}+V_{C}\left(\boldsymbol{r}_{i}\right) \\
x_{\mathrm{rec}}=\frac{\boldsymbol{p}_{1} \cdot \boldsymbol{p}_{2}}{m_{C}} \quad(\text { recoil term })
\end{gathered}
$$

where $i=1$ (2) indicates the first (second) valence nucleon. The single-particle (SP) Hamiltonian, $h_{C}$, contains the mass parameters, $\mu_{i}=m_{N} m_{C} /\left(m_{N}+m_{C}\right)$, $m_{N}=939.565(938.272) \mathrm{MeV} / c^{2}$ for neutrons (protons), and $m_{C}$ of the core nucleus. Note that the recoil term also exists after the center-of-mass motion is subtracted. The SP potential, $V_{C}$, and the pairing potential, $v_{N N}$, are determined in the next section.

The SP state, $\psi_{n l j m}(\boldsymbol{r})=R_{n l j}(r) \cdot \mathcal{Y}_{l j m}(\theta, \phi)$, is solved to satisfy

$$
h_{C}(i) \psi_{n_{i} l_{i} j_{i} m_{i}}\left(\boldsymbol{r}_{i}\right)=e_{i} \psi_{n_{i} l_{i} j_{i} m_{i}}\left(\boldsymbol{r}_{i}\right)
$$

We employ the SP states up to the $h_{11 / 2}$ channel. In order to take into account the Pauli principle, we exclude the states occupied by the core. Continuum states are discretized within a box, $R_{\text {box }}=30 \mathrm{fm}$. The cutoff energy, $E_{\text {cut }}=30 \mathrm{MeV}$, is also employed to truncate the model space. We checked that this truncation indeed provides a sufficient convergence for results in the following sections.

For diagonalization of the total Hamiltonian, we employ the anti-symmetrized two-particle (TP) states for basis. That is, $\tilde{\Psi}_{k_{1} k_{2}}^{(J, M)}\left(\boldsymbol{r}_{1}, \boldsymbol{r}_{2}\right) \equiv \hat{\mathcal{A}} \Psi_{k_{1} k_{2}}^{(J, M)}\left(\boldsymbol{r}_{1}, \boldsymbol{r}_{2}\right)$, where

$$
\begin{aligned}
& \Psi_{k_{1} k_{2}}^{(J, M)}\left(\boldsymbol{r}_{1}, \boldsymbol{r}_{2}\right) \\
& \equiv \sum_{m_{1} m_{2}} \mathcal{C}_{m_{1} m_{2}}^{(J, M) j_{1} j_{2}} \psi_{n_{1} l_{1} j_{1} m_{1}}\left(\boldsymbol{r}_{1}\right) \psi_{n_{2} l_{2} j_{2} m_{2}}\left(\boldsymbol{r}_{2}\right)
\end{aligned}
$$

Here we take the short-hand label, $k_{i} \equiv\left\{n_{i} l_{i} j_{i}\right\}$.

The M1 operator, which operates only on the two valence nucleons, reads

$$
\hat{\mathcal{Q}}_{\nu}=\hat{q}_{\nu}(1)+\hat{q}_{\nu}(2)
$$

where

$$
\begin{gathered}
\hat{q}_{\nu=0}=\mu_{\mathrm{N}} \sqrt{\frac{3}{4 \pi}}\left(g_{l} \hat{l}_{0}+g_{s} \hat{s}_{0}\right), \\
\hat{q}_{\nu= \pm 1}=(\mp) \mu_{\mathrm{N}} \sqrt{\frac{3}{4 \pi}}\left(g_{l} \hat{l}_{ \pm}+g_{s} \hat{s}_{ \pm}\right) .
\end{gathered}
$$

Notice that $\hat{l}_{0}=\hat{l}_{z}, \hat{l}_{ \pm}=\left(\hat{l}_{x} \pm i \hat{l}_{y}\right) / \sqrt{2}$, and similarly for the spin operators. As well known, $g$ factors are given as $g_{l}=1(0)$, and $g_{s}=5.586(-3.826)$ for the proton (neutron) [35, 36]. In the following, we omit the nuclear magneton $\mu_{N}$ and the factor $\sqrt{3 / 4 \pi}$, except when it needs.

In this work, we discuss up to the one-body-operator level of the M1 excitation, whereas the meson-exchangecurrent effect is not included. In some ab-initio calculations, it has been shown that this effect indeed can contribute in addition to the M1 transition of the onebody-operator level [64-66]. For treatment of this effect, one needs to consider the relevant multi-body terms. This is, however, technically demanding, and beyond the scope of this work. We notify that evaluation of this meson-exchange-current effect is awaiting for the future progress.

\section{A. M1 sum rule}

The sum-rule value (SRV) of M1 transitions is determined as

$$
\begin{aligned}
S_{\mathrm{M} 1} \equiv & \sum_{f}\left(\left|\left\langle f\left|\hat{\mathcal{Q}}_{0}\right| i\right\rangle\right|^{2}\right. \\
& \left.+\left|\left\langle f\left|\hat{\mathcal{Q}}_{+}\right| i\right\rangle\right|^{2}+\left|\left\langle f\left|\hat{\mathcal{Q}}_{-}\right| i\right\rangle\right|^{2}\right),
\end{aligned}
$$


where $|i\rangle$ and $|f\rangle$ indicate the initial and final states, respectively. Within the three-body model, because only two nucleons are available to excite, this can be reduced to

$$
\begin{aligned}
S_{\mathrm{M} 1}= & \left\langle i\left|\left(g_{l} \hat{\mathbf{L}}_{12}+g_{s} \hat{\mathbf{S}}_{12}\right)^{2}\right| i\right\rangle \\
= & \left(g_{l}^{2}-g_{l} g_{s}\right)\left\langle\hat{\mathbf{L}}_{12}^{2}\right\rangle^{(i)}+\left(g_{s}^{2}-g_{l} g_{s}\right)\left\langle\hat{\mathbf{S}}_{12}^{2}\right\rangle^{(i)} \\
& +g_{l} g_{s}\left\langle\hat{\mathbf{J}}^{2}\right\rangle^{(i)}
\end{aligned}
$$

where $\hat{\mathbf{J}}=\hat{\mathbf{L}}_{12}+\hat{\mathbf{S}}_{12}, \hat{\mathbf{L}}_{12}=\hat{\mathbf{l}}(1)+\hat{\mathbf{l}}(2)$, and $\hat{\mathbf{S}}_{12}=\hat{\mathbf{s}}(1)+$ $\hat{\mathbf{s}}(2)$. Namely, the SRV contains the information on the coupled spins of the valence nucleons at the initial state. Considering the simplification of this M1 sum rule, there are two cases, which can be especially worth mentioning.

- For two neutrons with $g_{l}=0$, SRV is simply determined from the initial spin-triplet component, $N_{S_{12}=1}^{(i)}$ :

$$
\begin{aligned}
& S_{\mathrm{M} 1}(2 \mathrm{n})=g_{s}^{2}\left\langle\hat{\mathbf{S}}_{12}^{2}\right\rangle^{(i)} \\
& =g_{s}^{2} \sum_{S_{12}=0,1} S_{12}\left(S_{12}+1\right) N_{S_{12}}^{(i)}=2 g_{s}^{2} N_{S_{12}=1}^{(i)} .
\end{aligned}
$$

Notice that this spin-triplet component itself stands independently of the final-state properties.

- When two protons or neutrons are coupled to $J_{i}=$ 0 at the initial state $|i\rangle$, Eq. (9) can be remarkably simplified. In this case, in terms of the $L S$ coupling scheme, the allowed components include $\left(L_{12}, S_{12}\right)=(1,1)$ and $(0,0)$, only. Thus,

$$
S_{\mathrm{M} 1}\left(J_{i}=0\right)=2\left(g_{l}-g_{s}\right)^{2} N_{\left(L_{12}=1, S_{12}=1\right)}^{(i)},
$$

where we have utilized that the first-component, $N_{\left(L_{12}=1, S_{12}=1\right)}^{(i)}$, can be identical to $N_{L_{12}=1}^{(i)}$ as well as $N_{S_{12}=1}^{(i)}$.

Notice that, in both cases, the M1 SRV is enhanced (suppressed) when the $S_{12}=1\left(S_{12}=0\right)$ component is dominant. Therefore, the maximum limit of SRV is $2\left(g_{l}-g_{s}\right)^{2}$ when $N_{S_{12}=1}^{(i)}=100 \%$.

\section{B. no-pairing SRV}

It is worthwhile to mention the SRV in one special case, where the non-diagonal terms in the Hamiltonian can be neglected: $H \rightarrow H_{\mathrm{NP}}=h_{C}(1)+h_{C}(2)$. In this case, the SRV can be analytically obtained. Because of the diagonal Hamiltonian, the initial state with the total angular momentum $J_{i}$ should be solved as the single set of coupled SP states. That is,

$$
|i\rangle=\left[\left|l_{1} j_{1}\right\rangle \otimes\left|l_{2} j_{2}\right\rangle\right]^{\left(J_{i}\right)}
$$

where $l_{k}$ and $j_{k}$ indicate the angle-quantum numbers of the $k$ th valence nucleon. Thus, Eq. (9) is represented as

$$
\begin{aligned}
& S_{\mathrm{M} 1, \mathrm{NP}}=\left(g_{l}^{2}-g_{l} g_{s}\right) \sum_{L_{12}} L_{12}\left(L_{12}+1\right) N_{L_{12}}^{\left(l_{1} j_{1}, l_{2} j_{2} ; J_{i}\right)} \\
& \quad+\left(g_{s}^{2}-g_{l} g_{s}\right) 2 N_{S_{12}=1}^{\left(l_{1} j_{1}, l_{2} j_{2} ; J_{i}\right)}+g_{l} g_{s} J_{i}\left(J_{i}+1\right),
\end{aligned}
$$

where $N_{X}^{\left(l_{1} j_{1}, l_{2} j_{2} ; J_{i}\right)}$ indicates the contribution of the $X$ component in this initial state. The analytic derivation of $N_{X}^{\left(l_{1} j_{1}, l_{2} j_{2} ; J_{i}\right)}$ for an arbitrary set of $\left(l_{1}, j_{1}, l_{2}, j_{2}, J_{i}\right)$ can be found in, e.g. textbook [67]. This no-pairing, analytic SRV can provide a solid standard to benchmark one's theoretical model, as well as its computational implementation.

\section{NUMERICAL RESULT}

\section{A. ${ }^{18} \mathrm{O}$ nucleus}

For numerical calculation, first we focus on the ${ }^{18} \mathrm{O}$ nucleus. Its core, ${ }^{16} \mathrm{O}$, is suitable to the rigid-core assumption: the lowest $1^{+}$level of ${ }^{16} \mathrm{O}$ locates at $13.6 \mathrm{MeV}$ [68], which is sufficiently higher than the energy region we consider in the following. Thus, the core excitation by the M1 can be well separated from that of the two valence neutrons, allowing us to use the three-body model.

\section{1. no-pairing case}

For simplicity, we first neglect the non-diagonal terms, $v_{N N}$ and $x_{\text {rec }}$ in Eq. (1), thus we deal with the no-pairing Hamiltonian, $H_{\mathrm{NP}}=h_{C}(1)+h_{C}(2)$.

In the first step, we need to constrain the core-neutron potential, $V_{C}$, for the SP states by considering the coreneutron subsystem, ${ }^{17} \mathrm{O}$. For this purpose, we employ the Woods-Saxon (WS) potential:

$$
\begin{aligned}
V_{C}(\boldsymbol{r}) & =V_{0} f(r)+U_{l s}(\mathbf{l} \cdot \mathbf{s}) \frac{1}{r} \frac{d f}{d r}, \\
f(r) & =\frac{1}{1+e^{\left(r-R_{0}\right) / a_{0}}},
\end{aligned}
$$

where $R_{0}=r_{0} \cdot A_{C}^{1 / 3}, A_{C}=16, r_{0}=1.25 \mathrm{fm}, a_{0}=0.65$ $\mathrm{fm}, V_{0}=-53.2 \mathrm{MeV}$, and $U_{l s}=22.1 \mathrm{MeV} \cdot \mathrm{fm}^{2}$. These parameters reproduce well the empirical SP energies of ${ }^{17} \mathrm{O}$, as shown in Table I. Note that, due to the Pauli principle, we exclude from our basis $1 s_{1 / 2}, 1 p_{3 / 2}$, and $1 p_{1 / 2}$ states, which are occupied by the core.

In the following, we move to the core plus two-neutron system, ${ }^{18} \mathrm{O}$. In the no-pairing case, the GS can be trivially solved as

$$
\Psi_{\mathrm{GS}}\left(\boldsymbol{r}_{1}, \boldsymbol{r}_{2}\right)=\hat{\mathcal{A}}\left[\psi_{1 d_{5 / 2}}\left(\boldsymbol{r}_{1}\right) \otimes \psi_{1 d_{5 / 2}}\left(\boldsymbol{r}_{2}\right)\right]^{\left(J_{i}=0\right)},
$$

which satisfies $H_{\mathrm{NP}}\left|\Psi_{\mathrm{GS}}\right\rangle=E_{\mathrm{GS}}\left|\Psi_{\mathrm{GS}}\right\rangle$ with $E_{\mathrm{GS}}=$ $2 e\left(1 d_{5 / 2}\right)$. Notice that, from Table I, this GS energy 

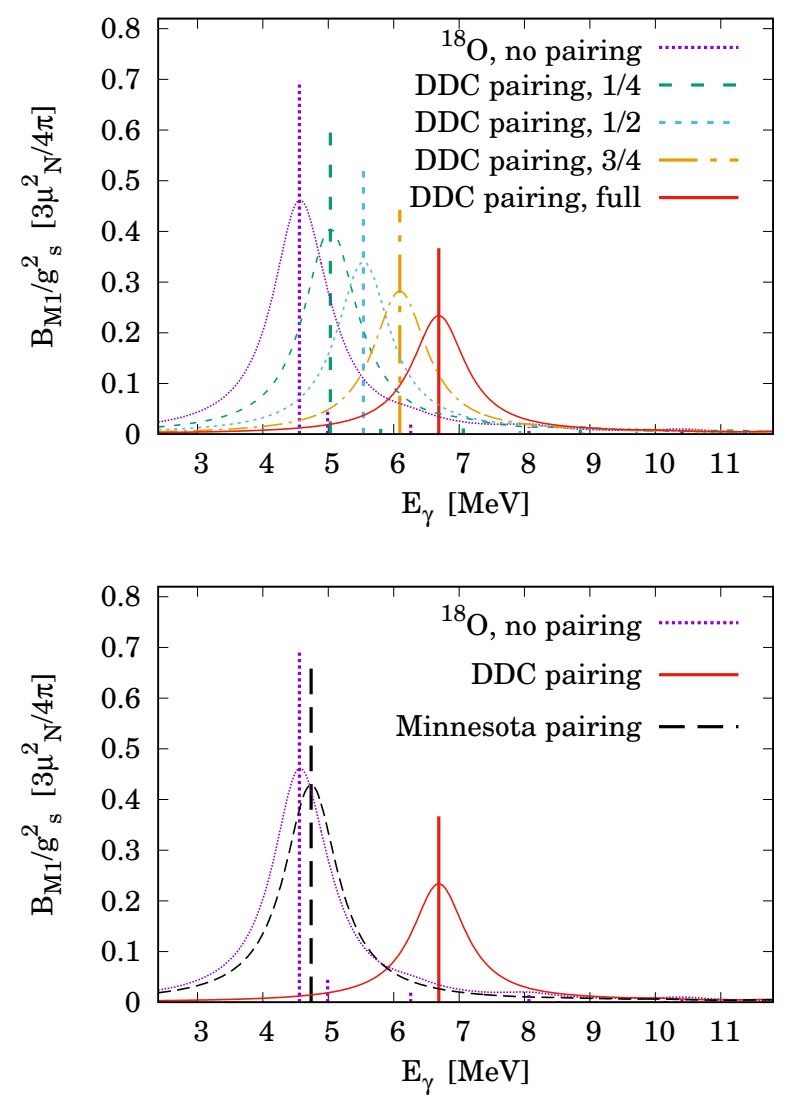

FIG. 1. Discrete M1 transition strength for ${ }^{18} \mathrm{O}$. Note that $E_{\gamma}=E_{f}-E_{\mathrm{GS}}$. The continuous distribution is also plotted by smearing the discrete strength with a Cauchy-Lorenz profile, whose full width at half maximum (FWHM) is $1.0 \mathrm{MeV}$.

is $2 e\left(1 d_{5 / 2}\right)=-8.286 \mathrm{MeV}$, which is higher than the empirical two-neutron binding energy, $B_{2 \mathrm{n}}=-12.188$ $\mathrm{MeV}$, of ${ }^{18} \mathrm{O}$ [68]. This discrepancy is, of course, due to the no-pairing assumption.

We now compute the M1 excitation from the $0^{+}$ ground state (GS). For $1^{+}$excited states, we solve all the TP states coupled to $1^{+}: H_{\mathrm{NP}}\left|f\left(1^{+}\right)\right\rangle=E_{f}\left|f\left(1^{+}\right)\right\rangle$.

TABLE I. Single-neutron energies for ${ }^{17} \mathrm{O}$ (in $\mathrm{MeV}$ ). For the resonant $d_{3 / 2}$ level, its energy and width $\Gamma$ are obtained by evaluating the scattering phase-shift.

\begin{tabular}{lccc}
\hline \hline & This work & Exp. [68] & Type \\
\hline$e\left(1 d_{5 / 2}\right)$ & -4.143 & -4.143 & bound \\
$e\left(2 s_{1 / 2}\right)$ & -3.275 & -3.272 & bound \\
$e_{\mathrm{r}}\left(d_{3 / 2}\right)$ & +0.902 & +0.941 & resonance \\
& $(\Gamma=0.102)$ & $(\Gamma=0.096)$ & \\
\hline \hline
\end{tabular}

In Fig. 1, we plot the M1 transition strength:

$$
B_{\mathrm{M} 1}\left(E_{\gamma}\right)=\sum_{\nu=0, \pm 1}\left|\left\langle f\left(1^{+}\right)\left|\hat{\mathcal{Q}}_{\nu}\right| \Psi_{\mathrm{GS}}\left(0^{+}\right)\right\rangle\right|^{2},
$$

where $E_{\gamma}=E_{f}-E_{\mathrm{GS}}$. Note that, because the system is spherical, $\nu=0$ and \pm 1 yield the same result. The figure also shows the continuous distribution obtained by smearing the discrete strength with a Cauchy-Lorenz profile, whose full width at half maximum (FWHM) is 1.0 MeV.

In Fig. 1, the highest transition strength without pairing locates at $E_{\gamma} \cong 4.6 \mathrm{MeV}$, which coincides well with the gap energy between the SP states $1 d_{5 / 2}$ and $1 d_{3 / 2}$. Thus, the transition $1 d_{5 / 2} \longrightarrow 1 d_{3 / 2}$ plays a major role in this case.

The numerical SRV can be obtained from the sum of discrete M1 transition strength, resulting in,

$$
S_{\mathrm{M} 1, \mathrm{cal} .}=\sum_{E_{\gamma}} B_{\mathrm{M} 1}\left(E_{\gamma}\right) \cong 0.799 g_{s}^{2} \text {. }
$$

On the other side, the corresponding analytic SRV for the $S_{12}=1$ component reads from Eq. (13),

$$
S_{\mathrm{M} 1, \mathrm{NP}}=2 g_{s}^{2} N_{S_{12}=1}^{\left(d_{5 / 2}, d_{5 / 2} ; J=0\right)},
$$

where the spin-triplet component $N_{S_{12}=1}^{\left(d_{5 / 2}, d_{5 / 2} ; J=0\right)}=2 / 5$ exactly [67], i.e. the analytic SRV equals $4 / 5 g_{s}^{2}=0.8 g_{s}^{2}$. Thus, our numerical SRV is consistent to the analytic one. Notice also that, in Fig. 1, the highest strength has $0.69 g_{s}^{2}$, which exhausts about $87 \%$ of the total SRV. This again means a major component comes from $1 d_{5 / 2} \longrightarrow$ $1 d_{3 / 2}$ transition.

\section{2. pairing case}

In the following, the non-diagonal terms of Hamiltonian, $v_{N N}$ and $x_{\text {rec }}$, are also taken into account in the analysis of ${ }^{18} \mathrm{O}$. For the pairing interaction, we employ a density-dependent contact (DDC) potential, similarly as in Refs. [28, 53, 55, 61, 62]. That is,

$$
\begin{aligned}
v_{N N}\left(\boldsymbol{r}_{1}, \boldsymbol{r}_{2}\right) & =w\left(\left|\boldsymbol{R}_{12}\right|\right) \cdot \delta\left(\boldsymbol{r}_{1}-\boldsymbol{r}_{2}\right), \\
w(r) & =w_{0}[1-f(r)],
\end{aligned}
$$

where $\boldsymbol{R}_{12}=\left(\boldsymbol{r}_{1}+\boldsymbol{r}_{2}\right) / 2$. The $f(r)$ is the same WS profile given in Eq. (14), and it schematically describes the density-dependence of the effective pairing interaction. Its bare strength is determined consistently from the neutron-neutron scattering length in vacuum, $a_{v}=$ $-18.5 \mathrm{fm}$, within the cutoff energy [61, 62]. That is,

$$
w_{0}=\frac{4 \pi^{2} \hbar^{2} a_{v}}{m_{n}\left(\pi-2 a_{v} k_{\text {cut }}\right)} \quad\left[\mathrm{MeV} \cdot \mathrm{fm}^{3}\right],
$$

where $k_{\text {cut }}=\sqrt{m_{n} E_{\text {cut }}} / \hbar$. 
TABLE II. Properties of the ground and $1^{+}$-excited states of ${ }^{18} \mathrm{O}$ obtained with the three-body-model calculation. Note that $E_{\mathrm{GS}}=-12.188 \mathrm{MeV}$ in the experimental data [68], with respect to the two-neutron-separation threshold. The sumrule values denoted by SRV are determined as $2 g_{s}^{2} N_{S_{12}=1}$, and shown in comparison to the calculated values $S_{\mathrm{M} 1 \text {,cal. }}$, which are obtained by integrating the $B_{\mathrm{M} 1}\left(E_{\gamma}\right)$ distribution.

\begin{tabular}{lrrr}
\hline \hline & DDC (full) & Minnesota & No pair. \\
\hline$E_{\mathrm{GS}}$ & $-12.019 \mathrm{MeV}$ & $-12.013 \mathrm{MeV}$ & $-8.286 \mathrm{MeV}$ \\
$\left\langle v_{N N}\right\rangle$ & $-4.337 \mathrm{MeV}$ & $-4.022 \mathrm{MeV}$ & $0 \mathrm{MeV}$ \\
$\left\langle x_{\mathrm{rec}}\right\rangle$ & $-0.177 \mathrm{MeV}$ & $-0.404 \mathrm{MeV}$ & $0 \mathrm{MeV}$ \\
$N_{d_{5 / 2} \otimes d_{5 / 2}}$ & $89.9 \%$ & $94.9 \%$ & $100 \%$ \\
$N_{S_{12}=1}$ & $19.7 \%$ & $34.9 \%$ & $40 \%$ \\
& (numerical) & (numerical) & (analytic) \\
$\mathrm{SRV}$ & $0.394 g_{s}^{2}$ & $0.698 g_{s}^{2}$ & $0.8 g_{s}^{2}$ \\
& & & \\
$E_{f}^{(1)}$ & $-5.329 \mathrm{MeV}$ & $-7.286 \mathrm{MeV}$ & $-3.729 \mathrm{MeV}$ \\
$S_{\mathrm{M} 1, \text { cal. }}$ & $0.393 g_{s}^{2}$ & $0.696 g_{s}^{2}$ & $0.799 g_{s}^{2}$ \\
\hline \hline
\end{tabular}

The two-neutron GS is solved by diagonalizing the three-body Hamiltonian via the anti-symmetrized TP basis coupled to $0^{+}$. As the result, the GS energy for ${ }^{18} \mathrm{O}$ is obtained, $E_{\mathrm{GS}}=-12.019 \mathrm{MeV}$, which is in fair agreement with the experimental value, $-12.188 \mathrm{MeV}$ [68]. On the other side, the $1^{+}$excited states, which satisfy $H\left|f\left(1^{+}\right)\right\rangle=E_{f}\left|f\left(1^{+}\right)\right\rangle$, are solved by diagonalizing the same Hamiltonian, but via the $1^{+} \mathrm{TP}$ basis.

In Table II, properties of the $0^{+}$ground state and the lowest $1^{+}$state are summarized. One can read that the pairing correlation leads to the deeper binding of two neutrons. Furthermore, the GS cannot be pure $\left(d_{5 / 2}\right)^{2}$ state, but it includes other components. As the result, the GS spin-triplet $\left(S_{12}=1\right)$ component is remarkably suppressed from the no-pairing case, similarly to the dineutron and diproton correlations $[28,55]$. Note that this component is evaluated by numerically integrating the initial-state density, $\left|\Psi_{\mathrm{GS}}\left(\boldsymbol{r}_{1}, \boldsymbol{r}_{2}\right)\right|^{2}$, but after the spintriplet projection. That is,

$$
N_{S_{12}=1}=\int d \boldsymbol{r}_{1} \int d \boldsymbol{r}_{2}\left|\hat{P}_{S_{12}=1} \Psi_{\mathrm{GS}}\left(\boldsymbol{r}_{1}, \boldsymbol{r}_{2}\right)\right|^{2} .
$$

Notice also that the total density is normalized as $\int d \boldsymbol{r}_{1} \int d \boldsymbol{r}_{2}\left|\Psi_{\mathrm{GS}}\left(\boldsymbol{r}_{1}, \boldsymbol{r}_{2}\right)\right|^{2}=N_{S_{12}=0}+N_{S_{12}=1}=1$.

In Fig. 1, the M1 transition strength with the DDC pairing interaction is presented for ${ }^{18} \mathrm{O}$. In comparison to the no-pairing case, a significant suppression of the transition strength is obtained. In Table II, the lowest $1^{+}$energy, $E_{f}^{(1)}$, is also displayed. Its change between the DDC and no-pairing cases is smaller than the corresponding change in the GS energy. Namely, compared with the GS energy, the excitation energies of $1^{+}$states are less sensitive on the DDC pairing interaction. Thus, the main M1 peak is shifted to the higher-energy region at $E_{\gamma} \cong 6.7 \mathrm{MeV}$.
In order to check the pairing effect more systematically, we repeat the same calculation but changing the absolute strength of the DDC-pairing interaction: $v_{N N} \longrightarrow$ $f \cdot v_{N N}$, where $f=0.25,0.50$, and 0.75 are employed. From the result shown in Fig. 1, one can clearly see that the M1 transition strength decreases with the increase of the pairing interaction. This is consistent to that the DDC-paring attraction enhances the spin-singlet component, which is not active for M1 excitation (for more details see the discussion in the following subsection). Also, because the energy level of the ground (excited) state is sensitive (insensitive) to the DDC interaction, the transition energy $E_{\gamma}$ increases when the attraction strength is enhanced.

The numerical sum-rule value can be obtained by integrating the strength distribution in Fig. 1. The result in the full-DDC case is

$$
S_{\mathrm{M} 1, \mathrm{cal} .}=\sum_{E_{\gamma}} B_{\mathrm{M} 1}\left(E_{\gamma}\right) \cong 0.393 g_{s}^{2}
$$

This value is, as expected from Eq. (10), consistent with the initial $S_{12}=1$ component, $S_{\mathrm{M} 1}(2 \mathrm{n})=2 g_{s}^{2} N_{S_{12}=1}$, where $N_{S_{12}=1}=0.197$ as evaluated by Eq. (20). Corresponding to the reduced M1 transition strength, the SRV is also suppressed by the DDC pairing correlation.

From the experimental side, there is no evidence of the low-lying $1^{+}$state in ${ }^{18} \mathrm{O}$ around $4-8 \mathrm{MeV}$, as predicted in Fig. 1. One possible reason is that our theoretical model does not quantitatively reproduce the $1^{+}$excited states. Especially, the $1^{+}$excitation energies may be sensitive to the pairing interaction model, which has, however, not been optimized specifically to this unnaturalparity transition case. One should mention that, from the experimental point of view, the M1 excitation of ${ }^{18} \mathrm{O}$ may be rather minor and behind the present measurability, because the number of valence neutrons is only two. This is in contrast to some nuclei $[5-7,17-23]$, where $10-20$ valence nucleons can contribute to the M1 transition, and make its response sufficiently strong. For ${ }^{18} \mathrm{O}$ with the minor M1 excitation, the selective detection of $1^{+}$states is possibly demanding. One should consider, e.g. the dominance of the $\mathrm{E} 1$ mode, and the competition with the E2 mode. To extract the pure information on the M1 process from existing data, further efforts may be necessary. We leave these issues for the future study.

\section{3. pairing-model dependence}

In the following we explore in more details the pairingmodel dependence of the M1 excitations. For this purpose, the DDC pairing interaction is replaced to the Minnesota interaction [69], which has been utilized in the 
similar three-body-model calculations [70-73]. That is,

$$
\begin{aligned}
& U_{\mathrm{MIN}}\left(\boldsymbol{r}_{1}, \boldsymbol{r}_{2}\right)=v_{r} \exp \left(\frac{-d^{2}}{2 q^{2}}\right) \\
& +v_{s} \exp \left(\frac{-d^{2}}{2 \kappa_{s}^{2} q^{2}}\right) \hat{P}_{S_{12}=0}+v_{t}\left(\frac{-d^{2}}{2 \kappa_{t}^{2} q^{2}}\right) \hat{P}_{S_{12}=1},
\end{aligned}
$$

where $d \equiv\left|\boldsymbol{r}_{1}-\boldsymbol{r}_{2}\right|, v_{r}=200 \mathrm{MeV}, v_{s}=-91.85 \mathrm{MeV}$, $v_{t}=-178 \mathrm{MeV}, q=0.5799 \mathrm{fm}, \kappa_{s}=1.788$, and $\kappa_{t}=1.525$, as given in the original paper [69]. Here we also assume $u=1$ for Eq. (9) in Ref. [69]. The first term indicates a repulsive core, whereas the second (third) term describes the attractive force in the spin-singlet (triplet) channel. For the present calculation of ${ }^{18} \mathrm{O}$, however, we need to use the $3 \%$ enhancement, $f=1.03$, in order to reproduce the two-neutron separation energy:

$$
v_{N N}\left(\boldsymbol{r}_{1}, \boldsymbol{r}_{2}\right)=f \cdot U_{\mathrm{MIN}}\left(\boldsymbol{r}_{1}, \boldsymbol{r}_{2}\right) .
$$

Note also that the core-neutron WS potential and the cutoff parameters are common to the previous case.

Before going to the result, we describe a comparison between the matrix elements of the DDC and Minnesota interactions. For our basis, anti-symmetrized TP states, $\left\{\left|\tilde{\Psi}_{k_{1} k_{2}}\left(J^{P}\right)\right\rangle\right\}$ coupled to the spin-parity $J^{P}=0^{+}$and $1^{+}$, are employed. This can be decomposed into the spinsinglet and triplet parts:

$$
\begin{aligned}
\left|\tilde{\Psi}_{k_{1} k_{2}}\left(J^{P}\right)\right\rangle= & \alpha_{k_{1} k_{2}}^{(0)}\left|\tilde{\Psi}_{k_{1} k_{2}}\left(J^{P}, S_{12}=0\right)\right\rangle \\
& +\alpha_{k_{1} k_{2}}^{(1)}\left|\tilde{\Psi}_{k_{1} k_{2}}\left(J^{P}, S_{12}=1\right)\right\rangle .
\end{aligned}
$$

Thus, the matrix element of the pairing interaction can also be decomposed as

$$
\begin{aligned}
& \left\langle\tilde{\Psi}_{k_{1}^{\prime} k_{2}^{\prime}}\left(J^{P}\right)\left|v_{N N}\right| \tilde{\Psi}_{k_{1} k_{2}}\left(J^{P}\right)\right\rangle \\
& =\alpha_{k_{1}^{\prime} k_{2}^{\prime}}^{(0) *} \alpha_{k_{1} k_{2}}^{(0)}\left\langle v_{N N}\right\rangle_{\left(J^{P}, S_{12}=0\right) k_{1}^{\prime} k_{2}^{\prime} k_{1} k_{2}} \\
& \quad+\alpha_{k_{1}^{\prime} k_{2}^{\prime}}^{(1) *} \alpha_{k_{1} k_{2}}^{(1)}\left\langle v_{N N}\right\rangle_{\left(J^{P}, S_{12}=1\right)} k_{1}^{\prime} k_{2}^{\prime} k_{1} k_{2}
\end{aligned}
$$

For the zero-range DDC pairing, $v_{N N} \propto \delta\left(\boldsymbol{r}_{2}-\boldsymbol{r}_{1}\right)$, only the $S_{12}=0\left(S_{12}=1\right)$ term survives for $J^{P}=0^{+}\left(1^{+}\right)$ [74]. Therefore, the zero-range attraction enhances only the $S_{12}=0\left(S_{12}=1\right)$ component in the $J^{P}=0^{+}\left(1^{+}\right)$ state. On the other side, if $v_{N N}$ has a finite range, both terms may become non-zero for $J^{P}=0^{+}$and $1^{+}$.

In Table II, our results with the Minnesota neutronneutron interaction are summarized. First, one can find that the spin-triplet component, $N_{S_{12}=1}$, in the GS is slightly changed from the no-pairing case, but not as much as in the DDC-pairing case. In coincidence, the numerical SRV, $S_{\mathrm{M} 1, \text { cal. }}$, has a larger value when compared with the DDC-pairing case. This difference between the two pairing models is understood by their ranges. Because the Minnesota model has a finite range, for the $0^{+}$ GS, it can contribute both in the $S_{12}=0$ and $S_{12}=1$ channels, whereas the zero-range DDC model only enhances the $S_{12}=0$ component.
Similarly to the GS result, the $1^{+}$excitation of ${ }^{18} \mathrm{O}$ depends on the choice of the pairing model: the lowest $1^{+}$energy, $E_{f}^{(1)}$, shows a remarkable difference between the DDC and Minnesota cases. This is again a result of the finite range of the Minnesota interaction. With a finite range, its matrix element for the $1^{+} \mathrm{TP}$ states can be larger than in the case of the zero-range DDC pairing.

In Fig. 1, the M1-transition strength by the Minnesota pairing is displayed, in comparison to the DDC pairing and no-pairing case. The result for the Minnesota pairing seems rather similar to that of the no-pairing case, in contrast to the DDC-pairing case. The transition energy, $E_{\gamma}=E_{f}-E_{\mathrm{GS}}$, is only slightly changed from the nopairing result, because both the GS and excited energies are shifted by the Minnesota interaction. The $B_{\mathrm{M} 1}$ value is slightly decreased, consistently to that the $S_{12}=1$ component in the GS is reduced by the Minnesota pairing.

Consequently, although the pairing models are equivalently fitted to reproduce the standard GS energy, it does not guarantee the same prediction for the M1 excitation. It suggests that the M1 excitation data can be good reference observables to optimize the existing pairing models. Further developments to observe the minor, low-lying M1 excitation are now on demand from this point of view.

\section{B. mirror nucleus ${ }^{18} \mathrm{Ne}$}

Next we investigate the M1 excitations of the mirror nucleus, ${ }^{18} \mathrm{Ne}$, as the ${ }^{16} \mathrm{O}$ core plus two valence protons with the Coulomb repulsion. Some parameters in the three-body model are revised to take the mass $m_{p}$, different $g$ factors, and Coulomb repulsion into account. For the Coulomb repulsion, the same procedure as in Refs. $[34,55]$ is utilized. That is, for the core-proton subsystem, we additionally employ the Coulomb potential of an uniformly-charged sphere. Also, for the proton-proton interaction, the factor $f=1.085$ is used for the DDC pairing in order to reproduce the empirical two-protonseparation energy. Namely, $v_{p p}=f \cdot v_{N N}+e^{2} /\left|\boldsymbol{r}_{2}-\boldsymbol{r}_{1}\right|$, combined with the Coulomb repulsion. Other parameters are kept unchanged from the ${ }^{18} \mathrm{O}$ case.

Our results are summarized in Fig. 2 and Table III. Indeed, one can observe a similar behavior of the M1 transition strength, i.e., it is suppressed when the DDC pairing correlation exists. This conclusion coincides with the reduction of the spin-triplet component in the GS by the zero-range DDC pairing, as shown in Table III.

Next we move to the Minnesota-pairing case: $v_{p p}=$ $f \cdot U_{\mathrm{MIN}}+e^{2} /\left|\boldsymbol{r}_{2}-\boldsymbol{r}_{1}\right|$, where $f=1.148$ in order to reproduce the two-proton-separation energy. Its results are also displayed in Fig. 2 and Table III. From the comparison, one can observe the similar tendency as in the ${ }^{18} \mathrm{O}$ case. Namely, the reduction of the $S_{12}=1$ component in the GS is smaller than that by the DDC pairing. In coincidence, the $B_{\mathrm{M} 1}$ distribution shows only slightly different shape than the no-pairing result. 
TABLE III. Same as Table II, but for ${ }^{18} \mathrm{Ne}$. Note that $E_{\mathrm{GS}}=$ $-4.523 \mathrm{MeV}$ in the experimental data [68], with respect to the two-proton-separation threshold.

\begin{tabular}{lrrr}
\hline \hline & DDC & Minnesota & No pair. \\
\hline$E_{\mathrm{GS}}$ & $-4.527 \mathrm{MeV}$ & $-4.524 \mathrm{MeV}$ & $-1.154 \mathrm{MeV}$ \\
$\left\langle v_{p p}\right\rangle$ & $-4.015 \mathrm{MeV}$ & $-3.683 \mathrm{MeV}$ & $0 \mathrm{MeV}$ \\
$\left\langle x_{\mathrm{rec}}\right\rangle$ & $-0.127 \mathrm{MeV}$ & $-0.352 \mathrm{MeV}$ & $0 \mathrm{MeV}$ \\
$N_{d_{5 / 2} \otimes d_{5 / 2}}$ & $88.5 \%$ & $93.5 \%$ & $100 \%$ \\
$N_{S_{12}=1}$ & $18.9 \%$ & $33.0 \%$ & $40 \%$ \\
& $($ numerical $)$ & $($ numerical $)$ & $($ analytic $)$ \\
SRV & $0.378\left(g_{l}-g_{s}\right)^{2}$ & $0.66\left(g_{l}-g_{s}\right)^{2}$ & $0.8\left(g_{l}-g_{s}\right)^{2}$ \\
& & & \\
$S_{\mathrm{M} 1, \text { cal. }}$ & $0.376\left(g_{l}-g_{s}\right)^{2}$ & $0.656\left(g_{l}-g_{s}\right)^{2}$ & $0.799\left(g_{l}-g_{s}\right)^{2}$ \\
\hline \hline
\end{tabular}

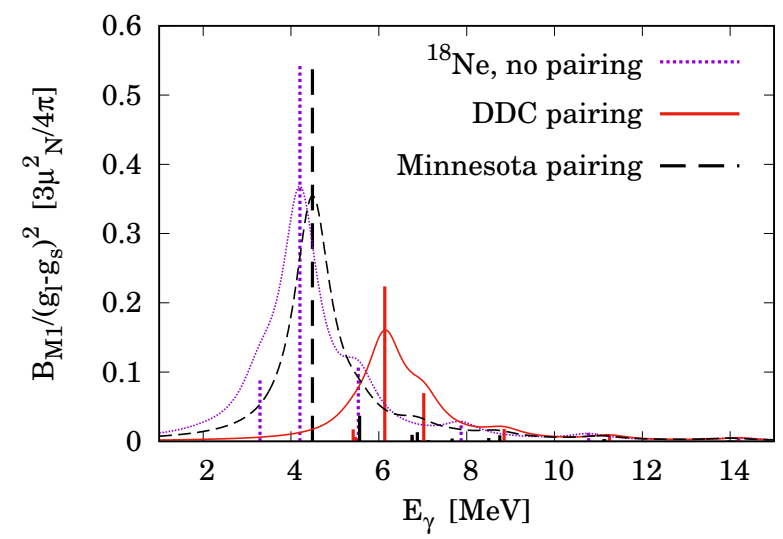

FIG. 2. Same as Fig. 1, but for ${ }^{18}$ Ne. Note that $E_{\gamma}=$ $E_{f}-E_{\mathrm{GS}}$.

The numerical SRVs in the DDC, Minnesota, and nopairing cases are obtained as $S_{\mathrm{M} 1 \text {,cal. }}=0.402\left(g_{l}-g_{s}\right)^{2}$, $0.656\left(g_{l}-g_{s}\right)^{2}$, and $0.799\left(g_{l}-g_{s}\right)^{2}$, respectively. Thus, the typical reduction factor between the pairing and nopairing SRVs is similar in the isobaric analogue systems, ${ }^{18} \mathrm{O}$ and ${ }^{18} \mathrm{Ne}$. We also note that the no-pairing SRV is consistent to the analytic solution, $4\left(g_{l}-g_{s}\right)^{2} / 5$.

Comparing with the ${ }^{18} \mathrm{O}$ result, ${ }^{18} \mathrm{Ne}$ shows a widely fragmented shape of the M1 strength. This is because the $1^{+}$state is not stable, but a resonance in the ${ }^{18} \mathrm{Ne}$ case. The typical (resonance) energy $E_{r}\left(1^{+}\right)$can be estimated from the mean transition energy $E_{\gamma}$ and the GS energy. From figures 1 \& 2 and tables II \& III, that is estimated, in e.g. the full-DDC pairing case, as $E_{r}\left(1^{+}\right) \cong+3.2(-5.3) \mathrm{MeV}$ for ${ }^{18} \mathrm{Ne}\left({ }^{18} \mathrm{O}\right)$. This energy difference is naturally understood by the Coulomb repulsive force both in the core-proton and proton-proton subsystems. Consequently, as a product of the Coulomb repulsion, the M1-excited state of ${ }^{18} \mathrm{Ne}$ may be unbound for the proton emission, and its M1 distribution becomes fragmented, originating from the non-zero width of the resonance state.

\section{C. $p f$-shell nucleus ${ }^{42} \mathbf{C a}$}

In the last two cases, we investigated the M1 excitation of the nucleon pair from the $s d$ shell. In the following, we move toward the $p f$-shell system, namely, ${ }^{42} \mathrm{Ca}$ with the ${ }^{40} \mathrm{Ca}$ core. For the ${ }^{40} \mathrm{Ca}$ nucleus, which is doubly shell-closed, there has been no $1^{+}$state measured in the low-lying region [68]. Thus, ${ }^{40} \mathrm{Ca}$ is suitable to our rigidcore assumption.

For numerical computation, we change some parameters as $A_{C}=40, V_{0}=-55.7 \mathrm{MeV}$, and $U_{l s}=10.8$ $\mathrm{MeVfm}^{2}$. Other parameters in $V_{C}, v_{N N}$, and cutoff parameters remain the same as in the ${ }^{18} \mathrm{O}$ case. This setting fairly reproduces the single-neutron energies of $1 f_{7 / 2}$ and $1 f_{5 / 2}$ in ${ }^{41} \mathrm{Ca}$ [68]. Due to the Pauli principle, we exclude the SP states up to $1 d_{3 / 2}$, which are occupied by the core.

The GS properties of ${ }^{42} \mathrm{Ca}$ are summarized in Table IV. Its GS energy obtained with the DDC or Minnesotapairing interaction is in a fair agreement with the empirical value, $-19.843 \mathrm{MeV}$ [68]. In the Minnesota case, the enhancement factor $f=1.12$ is needed to reproduce the GS energy: $v_{N N}=f \cdot U_{\mathrm{MIN}}$.

In Fig. 3, the M1 transition strength for ${ }^{42} \mathrm{Ca}$ is shown for all the cases. First, comparing the DDC and nopairing cases, one can find qualitatively the same conclusion as in the $s d$-shell nuclei: the DDC pairing suppresses the strength of M1 transitions. The SRV with the pairing is obtained as $S_{\mathrm{M} 1 \text {,cal. }}=0.352 g_{s}^{2}$. This result is, as expected from Eq. (10), consistent to the $S_{\mathrm{M} 1}(2 \mathrm{n})=2 g_{s}^{2} N_{S_{12}=1}$, where $N_{S_{12}=1}=0.176$ as shown in Table IV. Similarly to the $s d$-shell case, the M1 SRV is shown to be linked with the coupled spin, which reflects the zero-range pairing effect.

In ${ }^{42} \mathrm{Ca}$, the Minnesota pairing provides a significant change from the other two cases. First the $S_{12}=1 \mathrm{com}-$ ponent in the GS is enhanced from the no-pairing result. This enhancement then leads to the increase of the $B_{\mathrm{M} 1}$ and $S_{\mathrm{M} 1 \text {,cal. values, which can be consistent to our }}$ sum-rule formulation. The $1^{+}$-excitation energy is remarkably decreased, and thus, the transition energy, $E_{\gamma}$, locates at the lowest value among the three cases. These effects are understood from the finite range of the Minnesota force, similarly as explained in the ${ }^{18} \mathrm{O}$ case. In this ${ }^{42} \mathrm{Ca}$ case, however, the difference between the DDC and Minnesota models gets more significant, because of the $p f$-shell. This model dependence exists even when the DDC and Minnesota models are both fitted to the same GS energy.

\section{SUMMARY}

We have investigated M1 excitations in the systems with two-valence nucleons in the framework of the three- 
TABLE IV. Same as Table II, but for ${ }^{42}$ Ca. Note that $E_{\mathrm{GS}}=$ $-19.843 \mathrm{MeV}$ in the experimental data [68], with respect to the two-neutron-separation threshold.

\begin{tabular}{lrrr}
\hline \hline & DDC & Minnesota & No pair. \\
\hline$E_{\mathrm{GS}}$ & $-19.232 \mathrm{MeV}$ & $-19.843 \mathrm{MeV}$ & $-16.795 \mathrm{MeV}$ \\
$\left\langle v_{N N}\right\rangle$ & $-2.999 \mathrm{MeV}$ & $-3.221 \mathrm{MeV}$ & $0 \mathrm{MeV}$ \\
$\left\langle x_{\mathrm{rec}}\right\rangle$ & $-0.005 \mathrm{MeV}$ & $-0.012 \mathrm{MeV}$ & $0 \mathrm{MeV}$ \\
$N_{S_{12}=1}$ & $17.6 \%$ & $50.6 \%$ & $42.9 \%$ \\
& (numerical) & $($ numerical) & (analytic) \\
$\mathrm{SRV}$ & $0.352 g_{s}^{2}$ & $1.012 g_{s}^{2}$ & $0.858 g_{s}^{2}$ \\
& & & \\
$E_{f}^{(1)}$ & $-15.389 \mathrm{MeV}$ & $-18.253 \mathrm{MeV}$ & $-14.299 \mathrm{MeV}$ \\
$S_{\mathrm{M} 1, \text { cal. }}$ & $0.352 g_{s}^{2}$ & $1.011 g_{s}^{2}$ & $0.857 g_{s}^{2}$ \\
\hline \hline
\end{tabular}

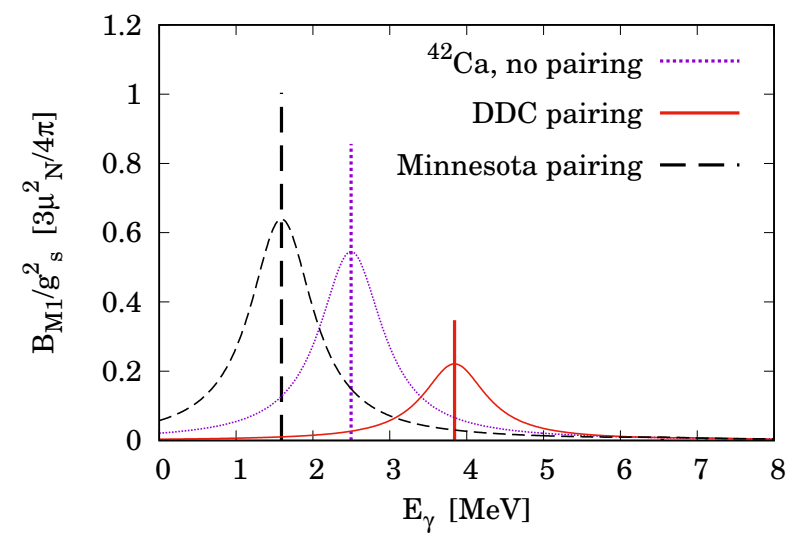

FIG. 3. Same as Fig. 1, but for ${ }^{42}$ Ca. Note that $E_{\gamma}=$ $E_{f}-E_{\mathrm{GS}}$.

body model. First we have introduced model independent M1 sum rule, that is applicable to nuclei with two protons or neutrons above the core with the shell closure. We showed that the total sum of M1 transition strength can be linked directly to the spin-triplet component of the two valence nucleons in the shell. We also performed the three-body model calculations of M1 tran- sition strength with the DDC and Minnesota-pairing interactions for ${ }^{18} \mathrm{O},{ }^{18} \mathrm{Ne}$, and ${ }^{42} \mathrm{Ca}$. Model calculations accurately reproduced the proposed M1 sum rule values.

It is shown that the M1 excitation can be sensitive to the choice of pairing model. The $B_{\mathrm{M} 1}$ and its SRV is enhanced or suppressed depending on the spin-triplet component in the GS, which depends on the pairing model. The same conclusion can apply both in the $s d$ and $p f$ shell nuclei. From these results, we expect that the M1 excitation is a promising probe to investigate the spin structure of valence nucleons, and/or to optimize the existing models for the pairing correlation.

One should notice that the meson-exchange-current effect on the M1 transition has not been taken into account in this work. This effect can provide additional components of the M1-excitation strength [64-66], which may enhance the M1 SRV. For evaluation of this effect, we need to expand our model calculation to take the twobody and/or more-body components into account. To evaluate this effect, especially for the nuclides which have been discussed within the present three-body model, is one remaining task for future.

From the experimental side, additional studies are necessary to extract the spin information from the M1 excitation data, in particular for the systems with twovalence nucleons above the closed-shell core, where the introduced M1 sum rule could be validated. The main problem is the contribution from other, electric modes, which lead to the hindrance against the M1 strength. A close collaboration between theory and experiment may be necessary to resolve this issue.

\section{ACKNOWLEDGMENTS}

T. Oishi sincerely thank T. O. Yamamoto and T. Uesaka for suggestions from the experimental side. This work is supported by the Croatian Science Foundation under the project Structure and Dynamics of Exotic Femtosystems (IP-2014-09-9159) and by the QuantiXLie Centre of Excellence, a project co financed by the Croatian Government and European Union through the European Regional Development Fund, the Competitiveness and Cohesion Operational Programme (KK.01.1.1.01).
[1] A. Richter, Progress in Particle and Nuclear Physics 13, 1 (1985).

[2] U. Kneissl, H. Pitz, and A. Zilges, Progress in Particle and Nuclear Physics 37, 349 (1996).

[3] N. Pietralla, P. von Brentano, and A. Lisetskiy, Progress in Particle and Nuclear Physics 60, 225 (2008).

[4] K. Heyde, P. von Neumann-Cosel, and A. Richter, Rev. Mod. Phys. 82, 2365 (2010), and references therein.

[5] D. Bohle, A. Richter, W. Steffen, A. Dieperink, N. L. Iudice, F. Palumbo, and O. Scholten, Physics Letters B 137, 27 (1984).
[6] D. Bohle, G. Kchler, A. Richter, and W. Steffen, Physics Letters B 148, 260 (1984).

[7] A. Richter, Nuclear Physics A 507, 99 (1990).

[8] T. Otsuka, Nuclear Physics A 507, 129 (1990).

[9] S. Kamerdzhiev and J. Speth, Nuclear Physics A 599, 373 (1996), proceedings of the Groningnen Conference on Giant Resonances.

[10] P. Vesely, J. Kvasil, V. O. Nesterenko, W. Kleinig, P. G. Reinhard, and V. Y. Ponomarev, Phys. Rev. C 80, 031302(R) (2009).

[11] V. O. Nesterenko, J. Kvasil, P. Vesely, W. Kleinig, P.-G. 
Reinhard, and V. Y. Ponomarev, Journal of Physics G: Nuclear and Particle Physics 37, 064034 (2010).

[12] V. O. Nesterenko, J. Kvasil, P. Vesely, W. Kleinig, and P.-G. Reinhard, Int. J. Mod. Phys. E 19, 558 (2010).

[13] R. Schwengner, S. Frauendorf, and B. A. Brown, Phys. Rev. Lett. 118, 092502 (2017).

[14] E. B. Balbutsev, I. V. Molodtsova, and P. Schuck, Phys. Rev. C 97, 044316 (2018).

[15] K. Langanke, G. Martínez-Pinedo, P. von NeumannCosel, and A. Richter, Phys. Rev. Lett. 93, 202501 (2004).

[16] J. Stone and P.-G. Reinhard, Progress in Particle and Nuclear Physics 58, 587 (2007).

[17] R. Moreh, S. Shlomo, and A. Wolf, Phys. Rev. C 2, 1144 (1970).

[18] R. M. Laszewski, P. Rullhusen, S. D. Hoblit, and S. F. LeBrun, Phys. Rev. Lett. 54, 530 (1985).

[19] R. M. Laszewski, R. Alarcon, D. S. Dale, and S. D. Hoblit, Phys. Rev. Lett. 61, 1710 (1988).

[20] S. Kamerdzhiev, J. Speth, G. Tertychny, and J. Wambach, Zeitschrift für Physik A 346, 253 (1993).

[21] R. Fearick, G. Hartung, K. Langanke, G. MartnezPinedo, P. von Neumann-Cosel, and A. Richter, Nuclear Physics A 727, 41 (2003).

[22] H. Pai, T. Beck, J. Beller, R. Beyer, M. Bhike, V. Derya, U. Gayer, J. Isaak, Krishichayan, J. Kvasil, B. Löher, V. O. Nesterenko, N. Pietralla, G. Martínez-Pinedo, L. Mertes, V. Y. Ponomarev, P.-G. Reinhard, A. Repko, P. C. Ries, C. Romig, D. Savran, R. Schwengner, W. Tornow, V. Werner, J. Wilhelmy, A. Zilges, and M. Zweidinger, Phys. Rev. C 93, 014318 (2016).

[23] T. Shizuma, T. Hayakawa, I. Daito, H. Ohgaki, S. Miyamoto, and F. Minato, Phys. Rev. C 96, 044316 (2017).

[24] D. Brink and R. Broglia, Nuclear Superfluidity: Pairing in Finite Systems, Cambridge Monographs on Particle Physics, Nuclear Physics and Cosmology (Cambridge University Press, Cambridge, UK, 2005).

[25] R. A. Broglia and V. Zelevinsky, eds., Fifty Years of Nuclear BCS: Pairing in Finite Systems (World Scientific, Singapore, 2013).

[26] D. J. Dean and M. Hjorth-Jensen, Rev. Mod. Phys. 75, 607 (2003).

[27] M. Bender, P.-H. Heenen, and P.-G. Reinhard, Rev. Mod. Phys. 75, 121 (2003).

[28] K. Hagino and H. Sagawa, Phys. Rev. C 72, 044321 (2005).

[29] K. Yoshida, Phys. Rev. C 80, 044324 (2009).

[30] J. Terasaki and J. Engel, Phys. Rev. C 84, 014332 (2011).

[31] I. Stetcu, A. Bulgac, P. Magierski, and K. J. Roche, Phys. Rev. C 84, 051309(R) (2011).

[32] S. Ebata, T. Nakatsukasa, and T. Inakura, Phys. Rev. C 90, 024303 (2014)

[33] N. D. Dang and N. Q. Hung, Journal of Physics G: Nuclear and Particle Physics 40, 105103 (2013).

[34] T. Oishi, K. Hagino, and H. Sagawa, Phys. Rev. C 84, 057301 (2011).

[35] J. Eisenber and W. Greiner, Nuclear Theory Volume 2: Excitation Mechanisms of the Nucleus (North-Holland Publishing Company, Amsterdam, 1970).

[36] P. Ring and P. Schuck, The Nuclear Many-Body Problems (Springer-Verlag, Berlin and Heidelberg, Germany, 1980).

[37] S. Wang, Phys. Rev. A 60, 262 (1999).
[38] Y. Lu and C. W. Johnson, Phys. Rev. C 97, 034330 (2018).

[39] K. Ikeda, S. Fujii, and J. I. Fujita, Phys. Lett. 3, 271 (1963).

[40] J. I. Fujita, S. Fujii, and K. Ikeda, Phys. Rev. 133, 549 (1964).

[41] C. W. Johnson, Phys. Lett. B 750, 72 (2015).

[42] N. Hinohara, M. Kortelainen, W. Nazarewicz, and E. Olsen, Phys. Rev. C 91, 044323 (2015).

[43] G. Bonasera, M. R. Anders, and S. Shlomo, Phys. Rev. C 98, 054316 (2018).

[44] U. Garg and G. Colo, Prog. Part. Nucl. Phys 101, 55 (2018).

[45] Y. K. Gupta, K. B. Howard, U. Garg, J. T. Matta, M. Şenyiğit, M. Itoh, S. Ando, T. Aoki, A. Uchiyama, S. Adachi, M. Fujiwara, C. Iwamoto, A. Tamii, H. Akimune, C. Kadono, Y. Matsuda, T. Nakahara, T. Furuno, T. Kawabata, M. Tsumura, M. N. Harakeh, and N. Kalantar-Nayestanaki, Phys. Rev. C 97, 064323 (2018).

[46] H. Sagawa and T. Uesaka, Phys. Rev. C 94, 064325 (2016).

[47] D. Gambacurta, F. Catara, M. Grasso, M. Sambataro, M. V. Andrés, and E. G. Lanza, Phys. Rev. C 93, 024309 (2016).

[48] A. Mason, R. Chatterjee, L. Fortunato, and A. Vitturi, The European Physical Journal A 39, 107 (2009).

[49] F. Osterfeld, Rev. Mod. Phys. 64, 491 (1992).

[50] M. Matsuo, K. Mizuyama, and Y. Serizawa, Phys. Rev. C 71, 064326 (2005).

[51] M. Matsuo, H. Shimoyama, and Y. Ootaki, Physica Scripta T150, 014024 (2012).

[52] C. A. Bertulani and M. S. Hussein, Phys. Rev. C 76, 051602(R) (2007).

[53] K. Hagino, H. Sagawa, J. Carbonell, and P. Schuck, Phys. Rev. Lett. 99, 022506 (2007).

[54] K. Hagino and H. Sagawa, Phys. Rev. C 89, 014331 (2014).

[55] T. Oishi, K. Hagino, and H. Sagawa, Phys. Rev. C 82, 024315 (2010), with erratum.

[56] T. Oishi, K. Hagino, and H. Sagawa, Phys. Rev. C 90, 034303 (2014).

[57] K. Hagino and H. Sagawa, Few-Body Systems 57, 185 (2016).

[58] Y. Kikuchi, K. Ogata, Y. Kubota, M. Sasano, and T. Uesaka, Progress of Theoretical and Experimental Physics 2016, 103D03 (2016).

[59] L. V. Grigorenko, J. S. Vaagen, and M. V. Zhukov, Phys. Rev. C 97, 034605 (2018), and references therein.

[60] Y. Suzuki and K. Ikeda, Phys. Rev. C 38, 410 (1988).

[61] G. Bertsch and H. Esbensen, Annals of Physics 209, 327 (1991).

[62] H. Esbensen, G. F. Bertsch, and K. Hencken, Phys. Rev. C 56, 3054 (1997).

[63] L. Fortunato, R. Chatterjee, J. Singh, and A. Vitturi, Phys. Rev. C 90, 064301 (2014).

[64] A. Richter, A. Weiss, O. Haüsser, and B. A. Brown, Phys. Rev. Lett. 65, 2519 (1990).

[65] L. E. Marcucci, M. Pervin, S. C. Pieper, R. Schiavilla, and R. B. Wiringa, Phys. Rev. C 78, 065501 (2008).

[66] S. Moraghe, J. Amaro, C. Garca-Recio, and A. Lallena, Nuclear Physics A 576, 553 (1994).

[67] A. R. Edmonds, Angular Momentum in Quantum Mechanics, Princeton Landmarks in Physics (Princeton Uni- 
versity Press, Princeton, USA, 1960).

[68] "Chart of Nuclides", National Nuclear Data Center (NNDC); http://www.nndc.bnl.gov/chart/.

[69] D. Thompson, M. Lemere, and Y. Tang, Nuclear Physics A 286, 53 (1977).

[70] T. Myo, K. Katō, S. Aoyama, and K. Ikeda, Phys. Rev. C 63, 054313 (2001).

[71] Y. Suzuki, H. Matsumura, and B. Abu-Ibrahim, Phys.
Rev. C 70, 051302(R) (2004).

[72] K. Hagino and H. Sagawa, Phys. Rev. C 75, 021301(R) (2007).

[73] T. Myo, Y. Kikuchi, H. Masui, and K. Kato, Progress in Particle and Nuclear Physics 79, 1 (2014).

[74] Y. Tanimura, K. Hagino, and H. Sagawa, Phys. Rev. C 86, 044331 (2012). 